\title{
Avaliação dos índices de suplementação de Vitamina A em uma Unidade Básica de Saúde em São Gonçalo do Amarante/RN
}

\author{
Evaluation of Vitamin A supplementation in a Basic Health Unit in São Gonçalo do Amarante/RN \\ Evaluación de la suplementación con Vitamina A en una Unidad Básica de Salud en São Gonçalo \\ do Amarante/RN
}

Recebido: 03/12/2021 | Revisado: 11/12/2021 | Aceito: 14/12/2021 | Publicado: 21/12/2021

\author{
Vanessa Rayane Vieira \\ ORCID: https://orcid.org/0000-0001-8893-2387 \\ Universidade do Estado do Rio Grande do Norte, Brasil \\ E-mail: rayane.nutri@gmail.com \\ Marco Aurelio M. Freire \\ ORCID: https://orcid.org/0000-0002-6483-0498 \\ Universidade do Estado do Rio Grande do Norte, Brasil \\ E-mail: freire.m@gmail.com
}

\begin{abstract}
Resumo
A vitamina A é um micronutriente lipossolúvel, sendo uma das vitaminas mais importantes. Suas megadoses ofertadas na infância são indispensáveis no desenvolvimento saudável dos infantes, em especial para a adequada maturação do sistema visual. O presente estudo tem como objetivo avaliar os índices de busca pela suplementação da Vitamina A na Unidade Básica de Saúde (UBS) Jardim Petrópolis, localizado na Cidade de São Gonçalo do Amarante, no Estado do Rio Grande do Norte. Pesquisa do tipo quantitativa, descritiva, retrospectiva, longitudinal e documental estruturada a partir da verificação de prontuários de acompanhamento depositados na UBS supracitada, realizada sob aprovação do Comitê de Ética em Pesquisa local (\#4.091.448). Os dados coletados foram tabulados e digitados, com os dados estatísticos sendo obtidos com o programa estatístico Prism 5.0. Os dados sócioeconômicos revelaram que a totalidade dos responsáveis inquiridos eram do sexo feminino, predominantemente jovens (faixa etária de 26-35 anos; 48,83\%), de cor/raça parda (69\%) e com renda mensal de 2 a 3 salários mínimos (59,52\%). No tocante à suplementação de vitamina A no período avaliado (2014/2019), a maior frequência foi observada em crianças do sexo feminino, com oscilação entre as diferentes idades. Campanhas de saúde fazem-se necessárias, de modo a esclarecer, junto aos responsáveis, a importância de estratégias nutricionais e de suplementação que garantam os níveis adequados desse composto em crianças em período crítico de desenvolvimento, uma vez que o impacto da suplementação inadequada poderá refletir no desenvolvimento dos infantes.
\end{abstract}

Palavras-chave: Vitamina A; Suplementação; Alteração visual; Infantes; Desenvolvimento.

\begin{abstract}
Vitamin A is a fat-soluble micronutrient, being one of the most important vitamins. Its megadoses offered in childhood are essential for the healthy growth of infants, especially concerning a proper maturation of the visual system. This study aims to evaluate the search for Vitamin A supplementation in the Jardim Petrópolis Basic Health Unit (UBS), located in the city of São Gonçalo do Amarante, in the state of Rio Grande do Norte. Quantitative, descriptive, retrospective, longitudinal and structured documental research based on the verification of monitoring records deposited at the aforementioned UBS, carried out under the approval of the local Research Ethics Committee (\#4.091.448). The collected data were tabulated and typed, with the statistical data being obtained with the Prism 5.0 software. The socioeconomic analysis revealed that all parents or guardians surveyed were female, predominantly young (aged 26-35 years; 48.83\%), of brown color/race (69\%) and with a monthly income of 2 to 3 minimum wages (59.52\%). Regarding vitamin A supplementation across the period evaluated (2014/2019), the highest frequency was observed in female children, with fluctuations among different ages. Health campaigns are necessary in order to clarify, with the legal responsible for the children, the importance of nutritional and supplementation strategies that ensure adequate levels of this compound in children in a critical period of development, since the impact of inadequate supplementation may reflect in the development of infants.
\end{abstract}

Keywords: Vitamin A; Supplementation; Visual alteration; Infants; Development.

\section{Resumen}

La vitamina A es un micronutriente soluble en grasa y es una de las vitaminas más importantes. Sus megadosis que se ofrecen en la infancia son fundamentales para el sano desarrollo del lactante, sobre todo para la adecuada maduración del sistema visual. Este estudio tiene como objetivo evaluar el índice de búsqueda de suplementación con vitamina A 
en la Unidad Básica de Salud (UBS) Jardim Petrópolis, ubicada en la ciudad de São Gonçalo do Amarante, en el estado de Rio Grande do Norte. Investigación documental cuantitativa, descriptiva, retrospectiva, longitudinal y estructurada basada en la verificación de los registros de seguimiento depositados en la UBS antes mencionada, realizada bajo la aprobación del Comité de Ética en Investigación local (\# 4.091.448). Los datos recopilados se tabularon y tipificaron, obteniéndose los datos estadísticos con el programa estadístico Prism 5.0. El análisis socioeconómico reveló que la totalidad de los padres o responsables legales consultados eran mujeres, predominantemente jóvenes (grupo de edad 26-35 años; 48,83\%), de color/raza marrón (69\%) y con un ingreso mensual de 2 a 3 salarios mínimos (59,52\%). En cuanto a la suplementación con vitamina A en el período evaluado (2014/2019), la mayor frecuencia se observó en niñas, con fluctuaciones entre diferentes edades. Las campañas de salud son necesarias para aclarar, con los responsables, la importancia de las estrategias nutricionales y de suplementación que aseguren niveles adecuados de este compuesto en los niños en un período crítico de desarrollo, ya que el impacto de una suplementación inadecuada puede reflejarse en el desarrollo de los infantes.

Palabras clave: Vitamina A; Suplementación; Alteración visual; Infantes; Desarollo.

\section{Introdução}

Durante o desenvolvimento do organismo e até a idade adulta os seres humanos necessitam manter uma nutrição adequada, que de modo final os permitem ter uma vida saudável e plena. Para tanto, a ingestão de nutrientes em quantidades adequadas é primordial, os quais são divididos em duas categorias: macro e micronutrientes. Os macronutrientes constituem a maior parte da dieta, garantindo o suporte energético e nutricional diário ao organismo, sendo classificados em três grupos: carboidratos, proteínas e lipídios (Cozzolino, 2020). Os micronutrientes, por sua vez, são compostos requeridos em pequenas quantidades pelo organismo, atuando como catalisadores de reações químicas, coenzimas, no processo de antioxidação e também como hormônios (Sarni et al., 2010; Pedraza \& Queiróz, 2011; Macedo et al., 2019; Vaz et al., 2017). Deste grupo fazem parte os minerais (fósforo, zinco, magnésio, ferro, cálcio, potássio, manganês, selênio) e as vitaminas. Esta última categoria pode ser subdividida, de acordo com sua solubilidade, em hidrossolúveis (vitaminas C e do complexo B) e lipossolúveis (vitaminas A, D, E e K) (Cozzolino, 2020).

A vitamina A, também denominada retinol, é uma das vitaminas mais importantes. É facilmente convertida no organismo humano em ácido retinóico (sua forma ativa), sendo encontrada em duas formas principais: all-trans retinoic acid (ATRA, o mais importante) e 9-cis retinoic acid (9-cis RA), armazenando-se principalmente no fígado (90\%) e no plasma ou soro (10\%) (Teixeira, 2012), sendo utilizada sempre que necessário. Assim, deve-se adquirir este micronutriente em quantidades adequadas diariamente através dos alimentos, uma vez que o organismo não é capaz de sintetizá-lo.

Sua função fisiológica mais conhecida está associada ao processo visual, fazendo parte do grupo prostético das opsinas (proteínas sensíveis à luz na retina), também atuando como reguladora e moduladora do crescimento e da diferenciação celular (Silva et al., 2007), bem como elemento regulador do sistema imunológico e combatendo infecções (Sarni et al., 2010), com sua deficiência sendo associada ao aumento da mortimorbidade especialmente em doenças infecciosas (Bates, 1995), além de estar associada à baixa estatura e baixo peso em infantes em fase pré-escolar (Bueno \& Czepielewski, 2007).

Dentre as deficiências de micronutrientes, a deficiência da vitamina A (DVA) ocupa o segundo posto, mesmo nos países desenvolvidos, ficando apenas atrás da deficiência de ferro. A DVA é definida pelo Ministério da Saúde (2013) como "uma situação na qual as concentrações dessa vitamina estão baixas e contribuem para a ocorrência de agravos à saúde, como diarreia e morbidades respiratórias". O retinol é encontrado em alimentos de origem animal e em um pequeno número de bactérias. Sua pré-formação se dá através dos carotenoides, substâncias lipossolúveis que são convertidas em vitamina A no organismo, estando presentes em vegetais, frutas e óleos, sendo necessária a ingestão simultânea de lipídios para que ocorra sua adequada absorção (Cozzolino, 2020). Um dos primeiros sintomas da deficiência da vitamina A é a cegueira noturna, caracterizando um importante problema de saúde pública, sendo de grande importância a prevenção desta anomalia em crianças de países em desenvolvimento (Bueno \& Czepielewski, 2007). Desta forma, esta carência deve ser tratada a tempo de 
impedir o desenvolvimento da síndrome ocular denominada xeroftalmia, que pode desencadear um quadro de cegueira irreversível (Diniz \& Santos, 2000; Souza \& Vilas Boas, 2002).

A suplementação de vitamina A é recomendada durante o período de gravidez para melhorar desfechos que incluem morbidade e mortalidade materna (Júnior \& Lemos, 2010). No último trimestre gestacional ocorre a transferência transparentaria, com os recém-nascidos prematuros não recebendo o suporte nutricional adequado de vitaminas e minerais, dentre eles a vitamina A (Gomes et al., 2005). Em contrapartida, o uso excessivo da suplementação pode se revelar tóxico (Cozzolino, 2020), suscitando controvérsias no uso da suplementação no período gestacional. Sintomas como náuseas, sonolência, visão turva, icterícia, anorexia, cefaleia, perda de cabelo, dor muscular e abdominal, fraqueza e alteração do estado mental são consideradas sequelas do uso excessivo da vitamina A (Júnior \& Lemos, 2010).

Estudos epidemiológicos realizados nas últimas décadas apontam um grave índice de hipovitaminose A, considerada um agravante na saúde pública em alguns locais do Brasil, em especial na Região Nordeste (Azevedo et al., 2010; Queiróz et al., 2013; Paula et al., 2014), sendo diagnosticada como a mais grave da América Latina (Cozzolino, 2020).

O programa de suplementação da vitamina A, desde sua criação através do Instituto Nacional de Alimentação e Nutrição (INAN), sofreu modificações ao longo dos anos até sua paralisação pela extinção do Instituto mantenedor em 1997, sendo retomado em 1999 pela Coordenação Geral da Política Nacional de Alimentação e Nutrição (CGPAN), sofrendo então algumas ampliações através do Programa Nacional de Suplementação de vitamina A (PNSVA), bem como garantindo aquisição das cápsulas através do Laboratório Oficial do Ministério da Saúde, os Farmanguinhos (Miranda et al., 2018).

Como já apontado, por se tratar de um problema definido como de saúde pública na Região Nordeste do país (Queiróz et al., 2013; Novaes et al., 2016), faz-se necessário caracterizar a busca pelos pais, junto aos postos de saúde pública, das dosagens complementares de vitamina A necessárias para um correto desenvolvimento dos infantes. Deste modo, o presente estudo objetiva avaliar os índices de busca da suplementação de vitamina A pelos responsáveis por crianças em estágio crítico de crescimento na Unidade Básica de Saúde Jardim Petrópolis, São Gonçalo do Amarante/RN, observando o índice de oferta por idade.

\section{Metodologia}

Pesquisa do tipo quantitativa, descritiva, retrospectiva, longitudinal e documental realizada a partir da verificação de prontuários depositados na Unidade Básica de Saúde (UBS) Jardim Petrópolis do Município de São Gonçalo do Amarante, no Estado do Rio Grande do Norte (RN) durante os meses de julho de 2020 e novembro de 2021, realizada sob aprovação do Comitê de Ética em Pesquisa local (número CAAE: 28817120.5.0000.5294, \#4.091.448). A cidade de São Gonçalo do Amarante, localizada na região metropolitana de Natal/RN, possui uma área de $249.1 \mathrm{~km}^{2}$, tendo uma população estimada de 87.700 habitantes no ano de 2017.

Como supracitado, o presente estudo foi realizado com base em uma pesquisa do tipo documental, que utiliza fontes primárias, definidas como dados ou informações que ainda não receberam tratamento científico ou analítico (Marconi \& Lakatos, 2019), consistindo em identificar e analisar os documentos com uma finalidade específica (Souza et al., 2011), os quais, segundo o conceito ampliado de documento proposto por Cellard (2008), correspondem a qualquer vestígio do passado, que pode ser utilizado como prova, tendo sido adotado de modo exitoso em estudos de ciências da saúde (Souza et al., 2011).

\subsection{Procedimentos e instrumentos de coleta de dados}

Os dados coletados foram oriundos de prontuários de atendimento de crianças entre 06 e 59 meses, que receberam as doses de vitamina A a cada semestre entre os anos de 2014 e 2019. As doses foram ofertadas de acordo com a faixa etária de 
cada infante, com bebês de 06 meses recebendo uma dose de 100.000 UI; já as crianças entre 12 e 59 meses foram suplementadas com doses de 200.000 UI.

O local de estudo apresenta cerca de 26.400 dados de crianças no período de 5 anos, dentre eles 10.560 referentes ao período de 2014 a 2019. Destes, foram excluídos dados rasurados ou que a escrita não estivesse legível, visto que se tratam de dados manuscritos.

A coleta de dados ocorreu em um ambiente reservado, cedido pela secretaria da UBS por avaliação de prontuários, definido como "o documento único, constituído de um conjunto de informações, sinais e imagens registradas, geradas com base em fatos, acontecimentos e situações sobre a saúde do paciente e a assistência a ele prestada, de caráter legal, sigiloso e científico" (Vasconcellos et al., 2008), sendo um documento individualizado que permanece armazenado na unidade hospitalar ou de saúde.

Por se tratar de um estudo de cunho documental a partir da verificação de prontuários, como forma de minimizar os riscos de quebra de sigilo, todas as informações sobre o sujeito foram totalmente preservadas de acordo com os princípios éticos, não havendo a identificação dos nomes dos participantes nos formulários, de modo a garantir seu anonimato. Além disso, o acesso aos prontuários foi limitado apenas ao tempo mínimo necessário, coletando-se a quantidade e qualidade das informações específicas para a pesquisa, evitando-se também a não violação e a integridade física dos documentos consultados.

Adicionalmente, foi realizada a aplicação de um questionário sócio econômico com os responsáveis, adaptado do formulário validado do Censo Demográfico do Instituto Brasileiro de Geografia e Estatística (IBGE) (IBGE, 2009), após os mesmos serem informados da natureza da pesquisa e atestarem sua participação através da assinatura do Termo de Consentimento Livre e Esclarecido (TCLE) seguindo a legislação vigente.

\subsection{Tabulação e análise de dados}

Os dados obtidos foram tabulados e os gráficos e tabelas foram organizados com o auxílio do programa Microsoft Excel $^{\circledR}$, sendo analisados no programa estatístico Prisma 5.0 (GraphPad Software Inc., La Jolla, CA, Estados Unidos).

\section{Resultados}

As UBSs de São Gonçalo do Amarante armazenam dados de crianças que receberam doses de suplementação da vitamina A em anos anteriores. Assim, foram coletados os dados entre outubro de 2014 a dezembro de 2019. Após a coleta, foi possível identificar 1789 prontuários de infantes que estiveram na unidade com o intuito de tomar a dose da suplementação no interlúdio supracitado.

Os dados associados à caracterização sóciodemográfica dos responsáveis $(n=42)$ pelos infantes que buscaram suplementação de vitamina A no intervalo investigado (2015/2019) indicaram que as mulheres corresponderam à totalidade da amostra $(\mathrm{n}=46 ; 100 \%)$, com faixa etária predominante de 26 a 35 anos $(\mathrm{n}=21 ; 48,83 \%)$, seguida pelas faixas de 15 a 25 anos $(\mathrm{n}=10 ; 23,8 \%)$, de 36 a 45 anos $(\mathrm{n}=8 ; 19,04 \%)$ e mais de 46 anos $(\mathrm{n}=3 ; 7,14 \%)$. Em relação à cor/raça professada, a cor parda foi a predominante $(n=29 ; 69 \%)$, seguida da branca $(n=8 ; 19 \%)$ e negra $(n=5 ; 12 \%)$. No tocante à renda mensal aproximada, a maioria relatou receber 2 a 3 salários mínimos ( $\mathrm{n}=25 ; 59,53 \%)$ (Tabela 1). 
Tabela 1. Aspectos socioeconômicos dos responsáveis $(\mathrm{N}=42)$ coletados nos meses de setembro a novembro de 2021 na UBS Jardim Petrópolis, São Gonçalo do Amarante/RN

\begin{tabular}{lc}
\hline Variável & $\mathbf{n}(\%)$ \\
\hline Gênero & $0(0)$ \\
Masculino & $42(100)$ \\
Feminino & \\
Faixa etária & $10(23,8)$ \\
15 a 25 anos & $21(48,83)$ \\
26 a 35 anos & $8(19,04)$ \\
36 a 45 anos & $3(7,14)$ \\
Mais de 46 anos & \\
Cor/raça & $8(19)$ \\
Branca & $29(69)$ \\
Parda & $5(12)$ \\
Preta & \\
Renda mensal (salário & \\
mínimo) & \\
0 a 1 & $0(0)$ \\
1 a 2 & $13(30,95)$ \\
2 a 3 & $25(59,52)$ \\
3 a 4 & $3(7,15)$ \\
4 a 5 & $1(2,38)$ \\
5 ou mais & $0(0)$ \\
& Fonte: Autores $(2021)$
\end{tabular}

As UBSs de São Gonçalo do Amarante armazenam dados de crianças que receberam doses de suplementação da vitamina A em anos anteriores. Assim, foi possível realizar a coleta de dados referente ao período compreendido entre outubro de 2014 a fevereiro de 2019. Após a coleta, foi possível identificar 1789 prontuários de infantes que estiveram na unidade com o intuito de tomar a dose da suplementação no interlúdio supracitado.

De acordo com o banco de dados da UBS, 57\% das informações $(n=150)$ corresponderam a infantes do sexo feminino, com os demais $43 \%(\mathrm{n}=113)$ sendo do sexo masculino no período 2014 e 2015, 57\% ( $\mathrm{n}=158)$ corresponderam a crianças do sexo feminino e $43 \%(n=119)$ do sexo masculino entre 2015 e 2016, 50,1\% ( $n=152)$ corresponderam a crianças do sexo feminino e 49,9\% ( $n=148)$ do sexo masculino entre os anos de 2016 e $2017,56 \%(n=245)$ corresponderam a infantes do sexo feminino e 44\% (n=193) do sexo masculino entre 2017 e 2018 e 52\% (n=263) corresponderam a crianças do sexo feminino e 48\% (n=248) do sexo masculino no período entre 2018 e 2019 (Figura 1), notando-se uma oscilação no número total de crianças ao longo dos períodos, que pode ser decorrente da saída dos responsáveis do município ou mesmo a não busca voluntária das doses de suplementação da vitamina A. É interessante notar também que ao longo de todo o período avaliado (2014/2019) a quantidade de crianças do sexo feminino foi sempre maior que aquela observada para infantes do sexo masculino, mesmo no período entre 2016 e 2017, onde os valores se aproximaram da igualdade percentual, refletindo assim uma tendência natural da população brasileira. 
Figura 1. Gráfico indicativo dos níveis de suplementação de vitamina A entre os anos de 2014 e 2019 (em \%) na UBS Jardim Petrópolis, São Gonçalo do Amarante/RN, comparando os infantes dos sexos masculino e feminino.

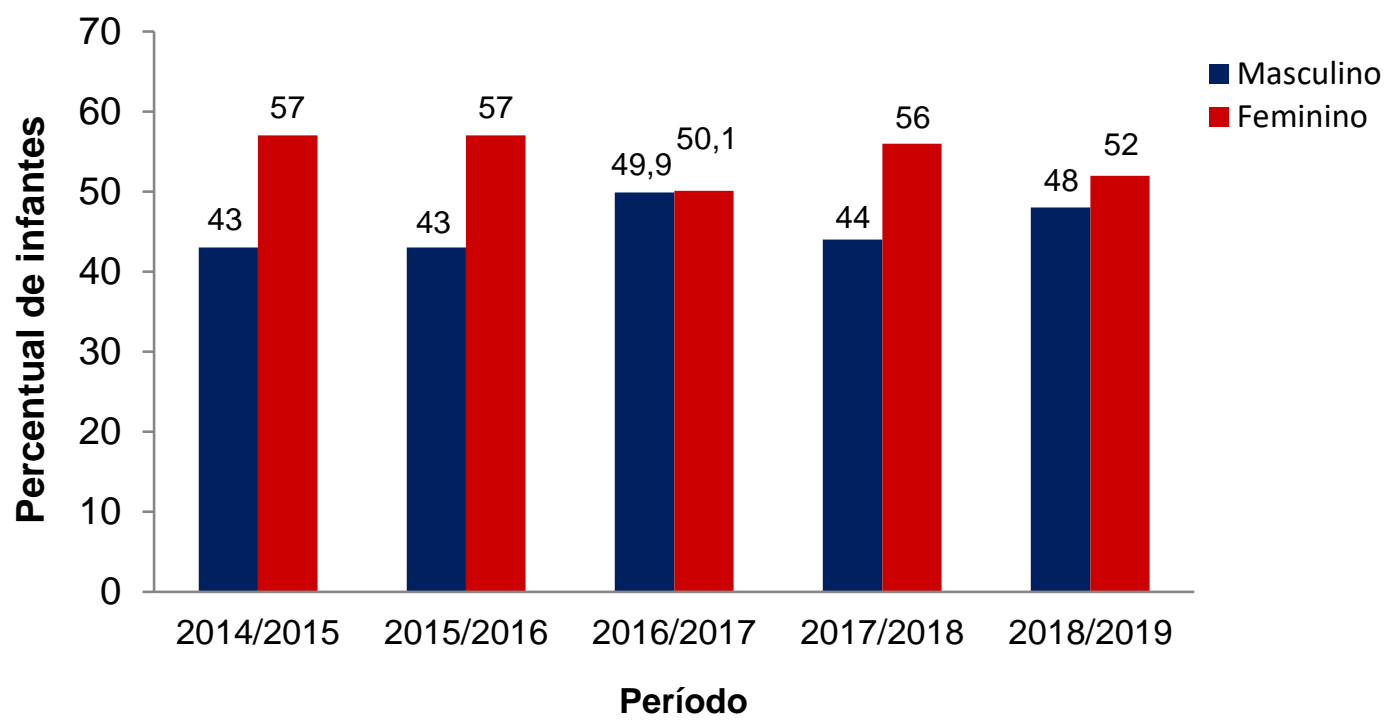

Fonte: Autores (2021).

Um dado peculiar a ser observado na Tabela 2 é a faixa etária inicial da administração das megadoses, que deveriam ser iniciadas no sexto mês de vida. No entanto, no período 2014/2015 apenas bebês do sexo feminino receberam a suplementação de 100.000 UI na idade correta (06 meses de idade), ocorrendo um atraso em relação aos bebês de sexo masculino, devido à falta de busca da suplementação pelos pais ou responsáveis.

O segundo menor percentual observado neste período localizou-se entre a faixa etária de 36 a 42 meses ( 3 anos a 3 anos e 06 meses). Vale ressaltar que para essa idade é administrada uma dose de 200.000UI de vitamina A - dose recomendada a partir de 12 meses - tanto para meninas $0,66 \%$ ( 1 criança), quanto para meninos $0,88 \%$ ( 1 criança) (Tabela 2).

Crianças do sexo feminino entre 18 a 24 meses ( 1 ano e 06 meses a 2 anos) apresentaram maior concentração no número de pré-escolares presente na suplementação no período 2014/2015, representando 22,66\% das meninas, seguido do grupo masculino com idades entre 30 a 36 meses ( 2 anos e 06 meses a 3 anos), com 21,23\% de presença (Tabela 2).

A Tabela 2 representa 277 que receberam as megadoses na UBS no período 2015/2016, sendo que 57\% delas eram do sexo feminino, e $43 \%$ do sexo masculino. Das 158 meninas, o maior percentual foi de infantes de 6 meses de vida, totalizando aproximadamente 13\% delas, e o menor na faixa etária de 36 a 42 meses de idade, totalizando 4,7\%. Em contrapartida, a maior incidência no grupo de meninos ocorreu entre 6 e 12 meses de idade (cerca de 13,7\%), sendo o menor índice das crianças entre 36 a 42 meses $(1,1 \%)$, seguido por bebês de 6 meses, na qual a busca pela primeira dose da vitamina foi de apenas $4,3 \%$.

Entre 2016 e 2017 houve um pequeno aumento na suplementação, onde 300 crianças foram registradas na sala de vacina, sendo a quantidade de meninos aproximadamente $1 \%$ menor que as meninas. Na Tabela 2 é perceptível um pequeno avanço na suplementação dos bebês de 6 meses do sexo masculino, representados por 7,3\% do total de infantes. O segundo maior percentual observado para este grupo ocorreu entre bebês de 6 meses a 1 ano de vida (cerca de 11\%), valor também observado no grupo feminino. Porém, na idade de 6 meses, a busca continuou mais elevada para o sexo feminino. O menor índice para ambos os grupos foi de 0,3\% para meninas e 0,6\% para meninos, na faixa etária de 36 a 42 meses. 
Nos anos mais recentes investigados (entre 2017 e 2019) observou-se um aumento importante na busca pelas megadoses de vitamina A. No período 2017/2018, tanto para bebês de 6 meses de idade quanto de 6 a 12 meses a taxa foi próxima de $11 \%$, bem como também no sexo masculino nesta mesma faixa etária. Já na idade de 6 meses foi possível observar que a busca pela primeira dose do suplemento, apesar de ainda ser considerada relativamente baixa, continuou evoluindo. Houve crescimento também nos anos de 2018 a 2019 em ambos os sexos, entre crianças de 36 a 42 meses (2\% para o sexo feminino e cerca de $4 \%$ para o sexo masculino) (Tabela 2).

Tabela 2. Descrição da busca por suplementação de vitamina A entre anos de 2014 e 2019 na UBS Jardim Petrópolis, São Gonçalo do Amarante/RN

\begin{tabular}{|c|c|c|c|c|c|c|c|c|c|c|c|}
\hline Sean & $\mathbf{N}$ & $\begin{array}{c}\text { Porcestagem } \\
\text { geral }\end{array}$ & $\begin{array}{l}\text { Faina etaria } \\
\text { (en meses) }\end{array}$ & $\mathbf{x}$ & $\begin{array}{c}\text { Porcentagem } \\
\text { por idade }\end{array}$ & Sese & $\mathrm{x}$ & $\begin{array}{c}\text { Percratapuin } \\
\text { Geral }\end{array}$ & $\begin{array}{l}\text { Faima etaria } \\
\text { (rmin meses) }\end{array}$ & $\mathbf{x}$ & $\begin{array}{l}\text { Porrentapen } \\
\text { Por idade }\end{array}$ \\
\hline \multirow[t]{8}{*}{ Feminine } & 150 & 376 & 06 & 24 & 100 & Fominaise & 152 & 30,15 & का & \$4 & $11, \pi$ \\
\hline & & & 21 & 14 & $21 \%$ & & & & $06 \times 12$ & 30 & 1035 \\
\hline & & & 21 & 14 & 218 & & & & 12 a 11 & 27 & 7,36 \\
\hline & & & 34 & 22 & $34 \%$ & & & & 18 a 24 & 22 & $7,29$. \\
\hline & & & 14 & 9 & $14 \%$ & & & & 24 a 30 & 16 & $5,7 \%$ \\
\hline & & & 17 & 11 & $17 \%$ & & & & 30.30 & 11 & $3, \pi$ \\
\hline & & & 1 & $t$ & 15 & & & & $36 \times 42$ & 2 & 0,05 \\
\hline & & & 18 & 12 & $18 \%$ & & & & $42 \times 48$ & 10 & $2.3 \%$ \\
\hline \multirow{8}{*}{ Masculine } & III & 4396 & क6 & 0 & 05 & Mtoventites & Ia & 79,15 & 86 & 22 & 7,55 \\
\hline & & & 06212 & 39 & $34 \%$ & & & & $05 \times 12$ & 34 & 11,34 \\
\hline & & & $12 \times 18$ & is & $13,27 \%$ & & & & 12 a 11 & 24 & 35 \\
\hline & & & $18 \times 24$ & 18 & $15,92 \%$ & & & & 152.24 & 26 & 2.75 \\
\hline & & & $24 \times 30$ & 3 & $2,65 \%$ & & & & $24 a 30$ & 14 & $4 \pi$ \\
\hline & & & 30236 & 24 & $21,23 \%$ & & & & 30.36 & 9 & is \\
\hline & & & 36142 & 1 & 0,8896 & & & & 36 a 42 & 1 & e.35 \\
\hline & & & $42 a 43$ & 13 & $11,50 \%$ & & & & $42 \times a$ & is & on \\
\hline Total & 263 & & & & 10006 & Total & 300 & & & & 10006 \\
\hline \multicolumn{6}{|c|}{$2014 / 2015$ - outubro a cutubro } & \multicolumn{6}{|c|}{20162017 - dezrmiteo a detrmitio } \\
\hline Sese & $\mathbf{N}$ & $\begin{array}{c}\text { Percentager } \\
\text { zeral }\end{array}$ & $\begin{array}{l}\text { Faisa velaria } \\
\text { (em merses) }\end{array}$ & N & $\begin{array}{c}\text { Porcesetageai } \\
\text { per itlade }\end{array}$ & Sexo & $\mathbf{N}$ & $\begin{array}{c}\text { Percentayem } \\
\text { ceral }\end{array}$ & $\begin{array}{l}\text { Fasa etaria } \\
\text { (ese meses) }\end{array}$ & N & $\begin{array}{l}\text { Porcentagem } \\
\text { per idade }\end{array}$ \\
\hline \multirow[t]{8}{*}{ Feminine } & t5s & 3796 & 06 & 30 & $12.9 \%$ & Veminiso & 2245 & 565 & 06 & II & $11,6 \%$ \\
\hline & & & $06 \times 12$ & 29 & 10,35 & & & & $06 \times 12$ & 47 & 10,746 \\
\hline & & & 12.16 & 23 & 1.34: & & & & $12 \mathrm{a11}$ & 4) & 9,946 \\
\hline & & & $18 \times 24$ & 16 & 9.846 & & & & 11.24 & 29 & 6,006 \\
\hline & & & $24 a 30$ & 14 & 146 & & & & 24230 & 24 & 5,946 \\
\hline & & & 30.36 & 12 & 4.35 & & & & $30 \times 16$ & 2n & 6.496 \\
\hline & & & 36.42 & 4 & $4,7 \%$ & & & & $36 \mathrm{a} 42$ & 6 & 1,3746 \\
\hline & & & $42 a 41$ & 34 & 1.75 & & & & 42,48 & 17 & 3,006 \\
\hline \multirow[t]{8}{*}{ Masculine } & 176 & 435 & 06 & 12 & 435 & Mtascutioe & $79)$ & कas & 06 & 29 & 6,65 \\
\hline & & & $06 \times 12$ & 38 & 13,76 & & & & $06 \times 12$ & it & 9,34 \\
\hline & & & 12 a II & 17 & 60 & & & & $12+11$ & 2a & $6,4 \% 6$ \\
\hline & & & 11924 & 19 & $6,5 \%$ & & & & 15.24 & 32 & 3,346 \\
\hline & & & $24: 90$ & 14 & 596 & & & & $24 \times 30$ & 11 & 2,546 \\
\hline & & & $30 \times 36$ & 9 & 3,296 & & & & $30 \times 30$ & 27 & 6,25 \\
\hline & & & $30 \times 42$ & 3 & 1,16 & & & & $36 x+2$ & $i$ & $1,3 \times 6$ \\
\hline & & & 42 a 411 & 7 & 1.44 & & & & 42.41 & 17 & 3.956 \\
\hline Total & 271 & & & & $100 \%$ & \multirow{2}{*}{\multicolumn{3}{|c|}{$2017 / 2018$ - jankiro o jantifo }} & & & 1006 \\
\hline \multirow{19}{*}{\multicolumn{3}{|c|}{20152016 - sovembro a novembro }} & & & & & & & & & \\
\hline & & & Sesen & $\mathbf{N}$ & $\begin{array}{c}\text { Poreentagen } \\
\text { geral }\end{array}$ & $\begin{array}{l}\text { Faisa otiria } \\
\text { (em meses) }\end{array}$ & $\mathbf{N}$ & $\begin{array}{c}\text { Porcentagem } \\
\text { por idade }\end{array}$ & & & \\
\hline & & & Ftminine & 269 & $52 \%$ & 66 & 55 & $10,7 \%$ & & & \\
\hline & & & & & & 06 a 12 & 59 & 11,55 & & & \\
\hline & & & & & & $12 \times 18$ & 52 & 105 & & & \\
\hline & & & & & & $18 \div 24$ & 21 & $4 \%$ & & & \\
\hline & & & & & & $24 \approx 30$ & 19 & 3,76 & & & \\
\hline & & & & & & $30 \times 36$ & 22 & 4,35 & & & \\
\hline & & & & & & 36 a 42 & 11 & 25 & & & \\
\hline & & & & & & 42 a 48 & 24 & 5,05 & & & \\
\hline & & & Masculine & 248 & $48 \%$ & 06 & 37 & 7,25 & & & \\
\hline & & & & & & $06 \times 12$ & 49 & $9,6 \%$ & & & \\
\hline & & & & & & $12=18$ & 38 & 7,45 & & & \\
\hline & & & & & & $18 \div 24$ & 19 & 3,756 & & & \\
\hline & & & & & & 24330 & 25 & $4.9 \%$ & & & \\
\hline & & & & & & $30 \times 36$ & 37 & 7,25 & & & \\
\hline & & & & & & $36 x+2$ & 20 & $3,95$. & & & \\
\hline & & & & & & $42 a+8$ & 23 & $4,5 \%$ & & & \\
\hline & & & Total & 511 & & & & $100 \%$ & & & \\
\hline
\end{tabular}

Fonte: Autores (2021). 


\section{Discussão}

Dados da Organização Mundial da Saúde (OMS) indicam que a DVA é um problema de saúde pública global, afetando ao redor de 19 milhões de mulheres grávidas e 190 milhões de crianças em idade pré-escolar, com regiões da África e Sudoeste da Ásia registrando os maiores índices (OMS, 2011).

Dentre as alterações decorrentes da ausência de vitamina A no organismo, a xeroftalmia é uma das mais importantes. Um dos primeiros indicativos dessa associação foi reportado por Venkataswamy e colaboradores (1976), que demonstraram que esta condição pode ser revertida com alimentos fontes de caroteno bem como com alimentos adicionados de retinol, com crianças com xeroftalmia considerada grave necessitando de vitamina A injetável ou na solução oral como forma de tratamento. Assim, um fator de grande importância a ser considerado na prevenção dessa anomalia em crianças é uma dieta adequada, com alimentos fonte deste nutriente, como por exemplo, vegetais folhosos verde-escuros, fígado, peixe, frutas amarelo-alaranjadas e ovo sendo ofertados. De acordo com Sommer e colaboradores (1980), crianças em idade pré-escolar apresentaram manchas de Bitot devido à deficiência de retinol, sendo considerado o fator mais importante para ocasionar essa alteração nos infantes, seguido da falta de amamentação.

Um ensaio clínico realizado na Indonésia com 88 crianças que apresentavam xeroftalmia comparou pacientes de dois grupos por cerca de 35 dias, com doses de 200.000 UI, com uma parte recebendo placebo (43 crianças) e outra recebendo vitamina A (45 crianças), considerando que as crianças que tinham xeroftalmia grave foram tratadas e não participaram do estudo, que inicialmente era composto por 118 participantes (Semba et al., 1990). Após avaliação clínica, constatou-se neste estudo que $73 \%$ das crianças que receberam a dose oral da vitamina A estavam completamente curadas e apenas $13 \%$ delas não obtiveram resposta ao tratamento. Neste mesmo estudo, constatou-se que a quantidade de mancha de Bitot presente nos olhos dos pacientes era diretamente relacionada com o nível sérico de vitamina A. Desta forma, quanto maior a quantidade de manchas nos olhos das crianças menor a disponibilidade do nutriente na corrente sanguínea, sendo detectáveis por exames laboratoriais. Além disso, um dado interessante foi o fato de que as crianças que não obtiveram resposta no tratamento eram do sexo masculino, bem como as maiores incidências da doença ocorreram entre os meninos (Semba et al., 1990). Em nossos achados, identificamos uma menor quantidade de infantes do sexo masculino recebendo a suplementação de vitamina A. Esse dado é preocupante, uma vez que, como citado, uma maior incidência das alterações visuais em decorrência da hipovitaminose A é mais elevada em crianças do sexo masculino.

$\mathrm{Na}$ mesma linha, um estudo realizado na Índia com 262 crianças entre 12 e 60 meses de idade, que apresentavam manchas de Bitot, aponta que 59\% desses infantes não receberam o colostro após o nascimento e $66 \%$ deles não receberam imunizantes contra o sarampo. O acompanhamento foi realizado em um período de 12 meses, sendo administrados $60 \mathrm{mg}$ de vitamina A no momento do diagnóstico e reforçada após 30 dias com a mesma dosagem, prosseguindo com a suplementação a cada 4/6 meses. Neste estudo um dos prognosticadores da doença eram crianças do sexo masculino. Após 3 meses do início da pesquisa, cerca 75\% das crianças estavam curadas e em 6 meses a cura das manchas de Bitot alcançou 95\% delas (Kapil et al., 2013).

Em um estudo realizado em Bangladesh Cohen e colaboradores (1987) constataram que a suplementação da vitamina A ocasionou $63 \%$ de proteção oftalmológica nas crianças acompanhadas, observando-se também que a prevalência das doenças oftalmológicas afetou mais meninos do que meninas. Avaliando-se a suplementação da vitamina A no Nepal, Katz e colaboradores (1995) analisaram dois grupos, com o primeiro com bebês de 1 a 11 meses e o segundo com crianças entre 12 e 56 meses. O estudo aponta que a dosagem da substância administrada interfere diretamente na resposta do tratamento, bem como também a quantidade administrada da vitamina. Embora novos casos de xeroftalmia tenham surgido nos infantes mesmo após o acompanhamento e administração do composto a cada 4 meses, pôde-se constatar que houve uma delimitação de $63 \%$ de novos casos, com suplementação de 200.000UI por criança 3 vezes ao ano. 
Ressaltando a importância da vitamina A administrada por via oral, alguns alimentos se constituem em fonte importante desse nutriente. O buriti, por exemplo, é um fruto regional presente especialmente no cerrado brasileiro, com sua polpa sendo considerada uma fonte de provitamina A. Seu fator nutritivo desencadeou a possibilidade de utilização no combate à xeroftalmia em crianças. Nesse sentido, um estudo feito no Brasil, avaliando a capacidade de cura da xeroftalmia em crianças através do consumo do doce do buriti nas regiões onde o fruto é de fácil acesso observou que em 36 das 44 crianças avaliadas que consumiram o doce por cerca de 20 dias tiveram respostas significativas e não apresentaram sinais de xeroftalmia, com apenas 6 das crianças apresentando sinais da doença, sem sintomas aparente (Mariath et al., 1989).

Milagres e colaboradores (2007) afirmam que os sintomas aparentes de xeroftalmia, manchas de Bitot e cegueira podem ser considerados determinantes de problema de saúde pública, bem como os exames oculares citológicos e os inquéritos alimentares, entrando também nessa classificação os indicadores séricos. Neste mesmo estudo, os autores afirmam ainda que uma avaliação feita com crianças no Estado da Bahia, aproximadamente 50\% das crianças avaliadas apresentou baixo índice de retinol sérico. Já no Estado do Rio de Janeiro cerca de 55\% dos recém-nascidos apresentou hipovitaminose A. Além disso, os bebês nascidos com baixo peso eram mais suscetíveis ao baixo índice de retinol sérico.

No Brasil, mesmo com a adoção de políticas de suplementação associadas às datas de vacinação (Brasil, 2013), a hipovitamimose de vitamina A ainda é considerada um problema de saúde pública nas regiões Norte e Nordeste do país (Queiróz et al., 2013; Novaes et al., 2016), desencadeada pela ausência desse composto em bebês e infantes pré-escolares, também sendo constatado baixo índice sérico em mulheres com idade reprodutiva e lactentes (Martins \& Mesquio, 2019). Elementos socioeconômicos, biológicos, maternos e alimentares foram apontados como fatores causais da deficiência do micronutriente, independente das famílias residirem em áreas rurais ou urbanas. No entanto, famílias com menor poder aquisitivo apresentam maior risco de desenvolverem algum tipo de deficiência nutricional, em especial a baixa do retinol sérico (Miglioli et al., 2013), o que se correlaciona com os dados do presente estudo, uma vez que a amostra avaliada na UBS de São Gonçalo do Amarante apresenta um poder aquisitivo não tão elevado (entre 2 e 3 salários mínimos).

Indicações a respeito dos benefícios da suplementação da vitamina $\mathrm{A}$ ainda são relativamente pouco acessíveis à população, em especial às famílias de baixa renda, visto que muitas crianças em idade escolar iniciam sua jornada de estudo com diagnóstico de desnutrição crônica, com um dos principais motivos considerados agravantes para a hipovitaminose A sendo o desmame precoce, bem como o baixo consumo de alimentos fontes do micronutriente, sendo o Nordeste Brasileiro considerado a região mais carente (Santos et al., 1996). Além disso, a suplementação com vitamina A em regiões endêmicas impacta em uma redução importante nos índices de mortalidade em infantes menores de cinco anos de idade (Beaton et al., 1993) em decorrência de uma melhoria significativa no estado nutricional da puérpera e do recém-nascido (Neves et al., 2015).

Muito se tem a entender sobre os motivos que levam a falta de busca pela suplementação de vitamina A, visto que de acordo com Paula e colaboradores (2010) no Nordeste brasileiro aproximadamente 17\% das crianças entre 6 e 59 meses apresentam deficiência desse composto. Em nossa pesquisa pôde-se comprovar que o menor índice de busca pela suplementação da vitamina A foi por responsáveis de bebês do sexo masculino na primeira dose, ou seja, aos 6 meses de vida, observando-se que no interlúdio 2014/2015 0\% dos infantes deste sexo foram suplementados na faixa etária, já comprovando o atraso e a possível perda de megadoses do micronutriente. Após os 48 meses de vida, caso a criança ainda tenha em sua caderneta de vacinação falta de suplementação, não será mais possível a oferta.

Uma das possíveis causas para justificar a evasão na busca pelas megadoses pode ser atribuída à falta de publicidade pelas autoridades públicas, e até mesmo de comunicação pelos profissionais de saúde nas UBS, observando que o menor índice de crianças suplementadas em todos os anos pesquisados e tanto para o sexo feminino e masculino, foi entre 36 e 42 meses, podendo ainda ser associado à caderneta de vacinação, pois nesta etária ocorre uma pausa nas vacinas, retornando aos 48 meses para as vacinas de reforço. Nos dados coletados, o menor índice de crianças de 3 a 3 anos e 6 meses foi do sexo 
feminino, representando 0,6\% do total no período 2016/2017. Já o maior percentual foi de 4,7\% no período 2015/2016, também do sexo feminino.

No presente estudo, ao serem indagados da importância da vitamina A como suplemento, alguns responsáveis indicaram apenas saber que é importante, mas sem compreensão do papel crucial no desenvolvimento de seus filhos. Desta forma, torna-se fundamental que campanhas de esclarecimento sejam realizadas, de modo a garantir o bem estar dos infantes, evitando-se o aparecimento de distúrbios oculares decorrentes de uma má alimentação ou falta de suplementação na dosagem correta.

\section{Considerações Finais}

A ausência ou quantidade insuficiente de vitamina A ainda persiste como um problema de saúde pública em nível global e, em âmbito nacional, notadamente na Região Nordeste do Brasil. Embora desde meados da década de 1990 os índices de suplementação tenham aumentado em decorrência do estabelecimento de uma política pública de associação da suplementação com as datas de vacinação, ainda há muito a progredir. Nesse sentido, campanhas de saúde fazem-se necessárias, de modo a esclarecer, junto aos responsáveis, a importância de estratégias nutricionais e de suplementação que garantam os níveis adequados de vitamina A em crianças em período crítico de desenvolvimento, uma vez que a ausência deste nutriente poderá se refletir no desenvolvimento dos infantes, causando especialmente alterações visuais importantes, além de impactar nos índices de mortalidade infantil.

À luz dos resultados do presente trabalho, mais estudos são necessários visando caracterizar os níveis de suplementação não apenas da vitamina A como de outros nutrientes durante o período crítico de desenvolvimento, de modo a proporcionar um crescimento saudável dos infantes.

\section{Agradecimentos}

Este estudo é parte dos requisitos para a obtenção do grau de Mestre em Saúde e Sociedade por Vanessa Rayane Vieira junto ao Programa de Pós-graduação em Saúde e Sociedade, Universidade do Estado do Rio Grande do Norte (UERN).

\section{Referências}

Bates, C. J. (1995). Vitamin A. Lancet, 345, 31-35. 10.1016/S0140-6736(95)91157-X

Beaton, G. H., Martorell, R., L'abbé, K. A., Edmonston, B., Mccabe, G., Rossi, A. C. \& Harvey, B. (1993). Effectiveness of Vitamin A Supplementation in the Control of Young Child Morbidity and Mortality in Developing Countries State-of-the-Art Series, Nutrition Policy Discussion Paper n. 13. Geneva: WHO, Administrative Committee Coordination/Subcommitee on Nutrition. https://www.unscn.org/layout/modules/resources/files/Policy_paper_No_13.pdf

Brasil. Ministério da Saúde. Secretaria de Atenção à Saúde. Departamento de Atenção Básica. Manual de condutas gerais do Programa Nacional de Suplementação de Vitamina A. Brasília: Ministério da Saúde, 2013. 34 p. https://www.saude.go.gov.br/images/imagens_migradas/upload/arquivos/201603/manual-de-condutas-gerais-do-programa-nacional-de-suplementacao-de-vitamina-a_20121.pdf

Bueno, A. L. \& Czepielewski, M. A. (2007). Micronutrientes envolvidos no crescimento. Revista HCPA Porto Alegre, 27(3), 47-56. http://hdl.handle.net/10183/164794

Cellard, A. (2008). A Análise Documental. In: Poupart, J. et al. (Orgs.). A pesquisa qualitativa: enfoques epistemológicos e metodológicos. Vozes, 295-316.

Cohen, N., Rahman, H., Mitra, M., Sprague, J., Islam, S., de Regt, E. L. \& Jali, M. A. (1987). Impact of massive doses of vitamin A on nutritional blindness in Bangladesh. American Journal of Clinical Nutrition, 45, 970-976. 10.1093/ajcn/45.5.970

Cozzolino, S.M.F. (2020). Biodisponibilidade de Nutrientes. (6a ed.) Manole; 960 p.

Diniz, A. S. \& Santos, L. M. P. (2000). Hipovitaminose A e xeroftalmia. Jornal de Pediatria, 76 (Supl.3), S311-S322. 0021-7557/00/76-Supl.3/S311

Gomes, M.M., Saunders, C. \& Accioly, E. (2000). Papel da vitamina A na prevenção do estresse oxidativo em recém-nascidos. Revista Brasileira de Saúde Materno Infantil, 5(3), 275-282. 10.1590/S1519-38292005000300003

IBGE. Instituto Brasileiro de Geografia e Estatística. Indicadores Sociodemográficos e de Saúde no Brasil. Número 25. IBGE; 2009. https://bit.ly/3DrZySB 
Júnior, H. P. L. \& Lemos, A. L. A. (2010). Vitamina A. Diagnóstico e Tratamento, 15(3), 122-124. http://files.bvs.br/upload/S/1413-9979/2010/v15n3/a1534.pdf

Kapil, U., Dwivedi, S., Sachdev, H. S., Dwivedi, S. N., Pandev R. M., Hirdyani, H. \& Upadhyay A. (2013). Resolution of Bitot's spots following mega-dose vitamin A supplementation in children between 1 and 5 years of age. 2013. Public Health Nutrition, 17(7), 1614-1619. 10.1017/S1368980013001584

Katz, J., West Jr, K. P., Khatry, S K., Thapa, M. D., LeClerq, S. C., Pradhan, E. K., Pokhrel, R. P. \& Sommer, A. (1995). Impact of vitamin A supplementation on prevalence and incidence of xerophthalmia in Nepal. Investigative Ophthalmology \& Visual Sciences, 36(13), 2577-2583. https://iovs.arvojournals.org/article.aspx?articleid=2179803

Macedo, C. N. A., Feitosa, J. M., Santos, M. R. M., Sousa, A. F. \& Duarte, E. C. P. S. (2019). Importância dos micronutrientes no desenvolvimento neurocognitivo da gestação a infância. Revista Uningá, 56(4), 145-155. http://revista.uninga.br/index.php/uninga/article/view/2985

Marconi, M. A. \& Lakatos, E. M. (2019). Fundamentos de Metodologia Científica. (8a ed.), Atlas.

Mariath, J. G. R., Lima, M. C. \& Santos, L. M. (1989). Vitamin A activity of buriti (Maurita vinifera Mart) and its effectiveness in the treatment and prevention of xerophthalmia. American Journal of Clinical Nutrition, 49(5), 849-853. 10.1093/ajcn/49.5.849

Martins, A. P. R. \& Masquio, D. C. L. (2019). O papel da vitamina A na saúde materno-fetal: uma revisão bibliográfica. Revista Eletrônica Acervo Saúde, (20), e518. 10.25248/reas.e518.2019

Miglioli, T. C., Fonseca, V. M., Junior, S. C. G., Lira, P. I. C. \& Batista Filho, M. (2013). Deficiência de Vitamina A em mães e filhos no Estado de Pernambuco. Ciência \& Saúde Coletiva, 18(5), 1427-1440. 10.1590/S1413-81232013000500028

Milagres, R. C. R. M., Nunes, L. C. \& Pinheiro-Sant'Ana, H. M. (2007). A deficiência de vitamina A em crianças no Brasil e no mundo. Ciência e Saúde Coletiva, 12(5), 1253-1266. 10.1590/S1413-81232007000500023

Miranda, W. D., Guimaraes, E. A. A., Campos, D. S. L, Antero, L. S., Beltão, N. R. M \& Luz, Z. M. P. (2018). Programa Nacional de Suplementação de Vitamina A no Brasil: um estudo de avaliabilidade. Revista Panamericana de Salud Publica, 42, e182. 10.26633/RPSP.2018.182

Neves, A. R., Saunders, C., Barros, D. C. \& Ramalho, A. (2015). Suplementação com vitamina A em gestantes e puérperas brasileiras: uma revisão sistemática. Revista Brasileira de Epidemiologia, 18(4), 824-836. 10.1590/1980-5497201500040012

Novaes, T. G., Gomes, A. T., Silveira, K. C., Souza C. L., Lamounier J. A., Netto, M. P., Capanema, F. D. \& Rocha, D. S. (2016). Prevalência e fatores associados com deficiência de vitamina A em crianças atendidas em creches públicas do Sudoeste da Bahia. Revista Brasileira de Saúde Materno Infantil, 16 (3), 345-352. 10.1590/1806-93042016000300007

OMS. Directriz: Administración de suplementos de vitamina A a lactantes y niños 6-59 meses de edad. Ginebra, Organización Mundial de la Salud, 2011.https://apps.who.int/iris/bitstream/handle/10665/44721/9789243501765_spa.pdf

Paula, W. K. A. S., Carminha, M. F. C., Figueirôa, J. N. \& Batista Filho, M. (2014). Anemia e deficiência de vitamina A em crianças menores de cinco anos assistidas pela Estratégia Saúde da Família no Estado de Pernambuco, Brasil. Ciência e Saúde Coletiva, 19(04), 1209-1222. 10.1590/141381232014194.00602013

Pedraza, D.F. \& Queiroz, D. (2011). Micronutrientes no crescimento e desenvolvimento infantil. Revista Brasileira de Crescimento e Desenvolvimento Humano, 21(1), 156-171. http://pepsic.bvsalud.org/pdf/rbcdh/v21n1/16.pdf

Queiróz, D., Paiva, A. A., Pedraza D. F., Cunha, M. A. L., Esteves, G. H., Luna, J. G. \& Diniz, A. S. (2013). Deficiência de vitamina A e fatores associados em crianças de áreas urbanas. Revista de Saúde Pública, 47(2), 248-256 10.1590/S0034-8910.2013047002906

Sarni, R. O. S, Souza, F. I. S., Cocco, R. R., Mallozi, M. C. \& Solé, D. (2010). Micronutrientes e sistema imunológico. Revista Brasileira de Alergia e Imunopatologia, 33(1), 8-13. http://www.sbai.org.br/revistas/Vol331/ART\%201-10\%20-\%20Micronutrientes\%20e\%20sistema\%20imunol\%C3\%B3gico.pdf

Santos, L. M. P., Filho, M. B. \& Diniz, A. S. (1996). Epidemiologia da carência de vitamina A no Nordeste do Brasil. Boletín de la Oficina Sanitaria Panamericana, 120 (6), 525-537. https://iris.paho.org/handle/10665.2/15460

Silva, S. L. V., Thiapó, A. P., Souza, G. G., Saunders, C. \& Ramalho, A. (2007). Micronutrientes na gestação e lactação. Revista Brasileira de Saúde Materno Infantil, 7(3), 237-244. 10.1590/S1519-38292007000300002

Semba, R. D., Wirasasmita, S., Natadisastra, G., Mulihal \& Sommer, M. A. (1990). Response of Bitot's spots in preschool children to vitamin A treatment. American Journal of Ophtalmology, 110(4), 416-420. 10.1016/s0002-9394(14)77024-2.

Sommer, A., Tarwotio, I., Muhilal, Djunaedi, E., \& Glover, J. (1980). Oral versus intramuscular vitamin a in the treatment of xerophthalmia. Lancet, 315 (8168), 557-559. 10.1016/S0140-6736(80)91053-3

Souza, J., Kantorski, L .P. \& Luiz, M. A. V. (2011). Análise documental e observação participante na pesquisa em saúde mental. Revista Baiana de Enfermagem, 25(2), 221-228. https://periodicos.ufba.br/index.php/enfermagem/article/view/5252/4469

Souza, W.A. \& Vilas Boas, O.M.G.C. (2002). A deficiência da vitamina A no Brasil: um panorama. Revista Panamericana de Salud Publica 12(3), 173-179. https://www.scielosp.org/pdf/rpsp/2002.v12n3/173-179/pt

Teixeira, R. A. (2010). Deficiência de Vitamina A e Fatores Associados em Crianças e adolescentes em Dois Municípios do Semiárido De Minas Gerais. Tese [Doutorado em Ciências da Saúde]. Faculdade de Medicina. Universidade Federal de Minas Gerais. 193 p. https://repositorio.ufmg.br/bitstream/1843/ECJS85SNM4/1/romero_alves_teixeira.pdf. Acesso em: 11 nov. 2021.

Vasconcellos, M. M., Gribel, E. B. \& Morais, I. H. S. (2008). Registros em saúde: avaliação da qualidade do prontuário do paciente, Rio de Janeiro, Brasil. Cadernos de Saúde Pública, 24 (Sup 1), S173-S182. 10.1590/S0102-311X2008001300021 
Research, Society and Development, v. 10, n. 17, e92101724302, 2021

(CC BY 4.0) | ISSN 2525-3409 | DOI: http://dx.doi.org/10.33448/rsd-v10i17.24302

Vaz, M. A., Oliveira, G. G., Pinheiro, M. S. \& Medeiros, E. F. F. (2017). Suplementação na infância e a prevenção da carência de micronutrientes: Artigo de revisão. Revista de Medicina e Saúde de Brasília, 6(1), 116-131. https://portalrevistas.ucb.br/index.php/rmsbr/article/view/7684

Venkataswamy, G., Krishnamurthy, K. A., Chandra, P., Kabir, S. A. \& Pirie, A. (1976). A nutrition rehabilitation centre for children with xerophthalmia. Lancet, 1(7969), 1120-1122. 10.1016/s0140-6736(76)99001-2 